\title{
NUMERICAL COMPUTATION OF THERMOELECTRIC EFFICIENCY OF GRAPHITE SHEET OPTIMAL DIMENSION
}

\author{
J. Dgheim*, A. Chahine, M. Ghazeleh, M. Abdallah \\ Laboratory of Applied Physics (LPA), Group of Mechanical, Thermal \& Renewable \\ Energies (GMTER)، Lebanese University, Faculty of Sciences II. \\ *Corresponding Author: J. Dgheim \\ jdgheim@ul.edu.lb
}

(Received August 2018- Accepted February 2019)

\begin{abstract}
J. Dgheim, A. Chahine, M. Ghazeleh, M. Abdallah. 2019. Numerical computation of thermoelectric efficiency of graphite sheet optimal dimension. Lebanese Science Journal. 20(1): 122-133.

Thermoelectric Graphite sheet has been studied numerically due to their ability to convert directly heat to electricity. Electro-thermal heat transfer equations coupling to initial and boundary conditions, are solved using finite difference and finite element schemes. The obtained results of both numerical techniques show good qualitative and quantitative agreements. In addition, the results of our numerical models present good conformity with the experimental result of Luo et al.. The voltage difference, the temperature variation, the Seebeck coefficient, the figure of merit and the maximum efficiency of graphite sheet are determined numerically. The ZT of the graphite sheet is calculated to be 1.27 at a temperature of $850 \mathrm{~K}$ for a graphite sheet surface of $0.26 \times 0.25 \mathrm{~cm}^{2}$.
\end{abstract}

Keywords: Thermoelectric, Seebeck coefficient, Heat transfer, Finite difference scheme, Finite element scheme.

\section{INTRODUCTION}

The challenges actually involve managing natural resource consumption, protecting soil and limiting greenhouse gas emissions. In this objective, thermoelectric materials offer interesting perspectives in order to generate electricity (Hochbaum et al., 
2008; Harman et al., 2002). Therefore, they offer the possibility to recover the deadly energies of industrial or domestic applications, and to develop refrigeration systems and heat pumps not requiring any coolant circulation. Moreover, thermoelectric materials can also produce electricity from waste heat sources to transform directly thermal energy into electricity. Finally, a quiet thermoelectric power converter can have a compact size.

The efficiency of the thermoelectric field is relatively classified as modest efficiencies in advanced sectors. However, the developments in the field of alloys and nanostructures have significantly increased the performance of the thermoelectric devices (Sootsman et al., 2009; Minnich et al., 2009).

The principle of thermoelectricity is associated to three fundamental effects. First, the Seebeck effect which is referred to be the thermo-power effect. The electrical potential is produced within a single conductor when a gradient of temperature is subjected. Then the Peltier effect, which is produced when a difference of temperature is created at the junctions of two different conductors while an electric current, is applied. Finally the Thomson effect, in which the heat contained in a single conductor is changed into a gradient of temperature while an electric current passes through it (Rowe, 1995; Radhakrishnan, 2008).

Excellent thermoelectric material should have a high electrical conductivity in order to reduce joule heating and a low thermal conductivity to avoid thermal shorting. In the same time, this material should also have a large Seebeck coefficient to maximize the conversion of heat to electrical power.

Many materials having low thermal conductivity have been investigated (Snyder et al., 2008; Kanatzidis, 2010; Gayner et al., 2016). In this objective, reducing the thermal conductivity improves the thermoelectric figure of merit coefficient. Kumar et al. (Kumar et al., 2013) developed a numerical model to study thermal and electrical energy transfer processes in a thermoelectric generator designed for waste heat recovery systems. Shi et al. (Shi et al., 2018) developed a two-dimensional finite volume model to investigate the performance of thermoelectric module. The voltage and temperature variations of the model under two boundary conditions (constant cold-side temperature and fixed convection heat transfer coefficient) are studied. A detailed study of thermoelectric properties of graphite pencil traces on a paper was carried out by Brus et al. (Brus et al., 2018) that measured the Hall and Seebeck effects.

Actually, special attention has been noticed to thermoelectric generation in order to recover energy. Thus, improved generation efficiency is required to commercialize thermoelectric materials with high performance. Therefore, the possibility of using 
graphite sheet as a thermoelectric material is considered in order to realize light thermoelectric modules from carbon.

The heat transfer in solid samples of Graphite sheet allows us to study the evolution of the temperature, taking into consideration the conduction and the electric transport in the materials. The measurement in this field requires very sophisticated experiments as it needs considerable affinity. A mathematical model describing the thermal and electrical transfers that take place in two-dimensional solid thermoelectric materials is proposed. This model is solved by two different methods: the explicit finite difference method and the finite element one. The thermal conductivity of the material has also a great influence on the transfer process. Graphite sheet is used as a thermoelectric material as it has many advantages, such as the light weight, the high selectivity of their shapes, the harmless nature and the low price. The results are presented in the form of linear temperature profiles, voltage, Seebeck coefficient, ZT coefficient of merit and efficiency.

\section{Mathematical Model}

The heat transfer phenomenon is the exchange of thermal energy that changes the internal energy of the physical systems. The rate of the heat transport depends on the temperatures of the systems, and the properties of the principal medium, through which the heat is transferred. Heat transfer occurs in a direction that increases the entropy of the system enhancing the Seebeck coefficient. Our mathematical model linked the heat transfer equation to Seebeck, Thomson, Joule and Peltier effects.

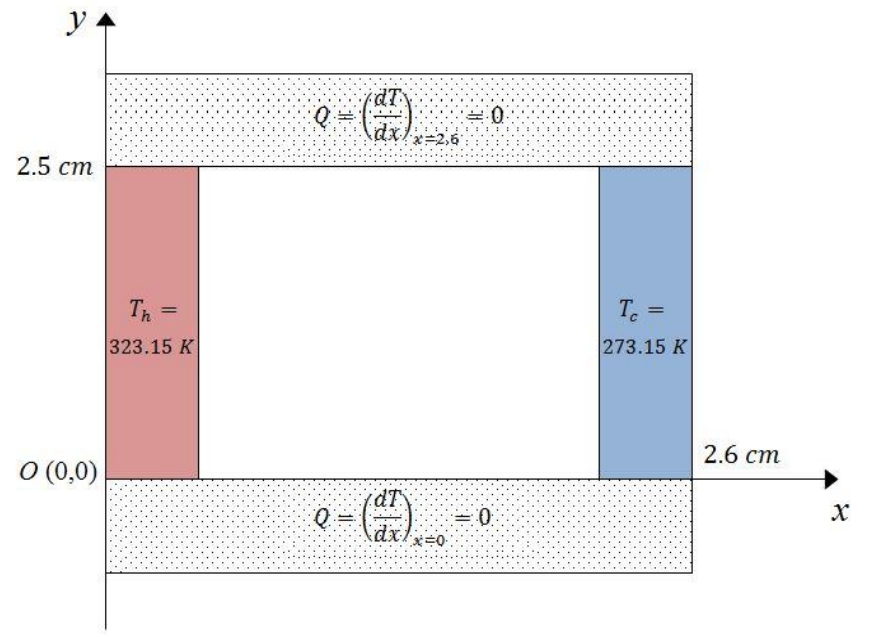

Figure 1. 2D Physical model of a thermoelectric solid. 
Figure 1 represents the physical model of a thermoelectric solid assimilated to a rectangular shape of two dimensions $(O x y)$. Two temperatures are applied on the edges of the rectangle, $T_{h}$ (hot temperature) and $T_{c}$ (cold temperature). The thermoelectric transfer equation is written as the following:

$$
\rho C_{p} \frac{\partial T}{\partial t}=\frac{\partial T}{\partial x}\left(K \frac{\partial T}{\partial x}\right)+\frac{\partial T}{\partial y}\left(K \frac{\partial T}{\partial y}\right)+\frac{J^{2}}{\sigma_{T}}-\tau J \nabla T
$$

Where $\rho$ is the density, $C_{p}$ is the specific heat at constant pressure, $T$ is the temperature, $t$ is the time, $K$ is the thermal conductivity, $\sigma_{T}$ is the electrical conductivity at constant temperature, $\tau$ is the Thomson coefficient and $J$ is the electrical density. The Thomson coefficient and the electrical density are both considered as variable in space and time.

The Peltier-Thomson term is the following: $T J \nabla \alpha$

Where $\square$ is the Seebeck coefficient.

Equation (2) contains both Thomson and Peltier contributions. By using the equivalence second relationship of Kelvin $\pi=\alpha T$, one can obtain:

$$
T J \nabla \alpha=T J \nabla \frac{\pi}{T}=T J\left[\frac{1}{T} \nabla \pi-\frac{1}{T^{2}} \pi \nabla T\right]=J[\nabla \pi-\alpha \nabla T]
$$

For pure Thomson effect:

$$
J[\nabla \pi-\alpha \nabla T]=J\left[\frac{d \pi}{d T}-\alpha\right] \nabla T=\tau J \nabla T
$$

With:

$$
\tau=\frac{d \pi}{d T}-\alpha
$$

The calculated efficiency is given by the figure of merit of a material as the following:

$$
Z T=\frac{\sigma \alpha^{2} T}{K}
$$

The efficiency coefficient is the following:

$$
\eta=\eta_{r, d} \frac{\Delta T}{T_{h}}=\frac{\sqrt{1+Z T}-1}{\sqrt{1+Z T}+\frac{T_{C}}{T_{h}}}
$$


Where $\eta_{r, d}$ is the reduced efficiency.

The relationship that links the two thermal $(K \square \square$ and electrical $(\sigma)$ conductivities given by Weidman Franz and reported by Luo et al. (Luo et al., 2002) is used:

$$
K=L \sigma T
$$

Where $L=2.14 \times 10^{-8} \mathrm{~W} . \mathrm{K}^{-2}$ is Lorenz number.

The Seebeck coefficient determined by Klein and Donadio (Klein an Donadio, 1967) is represented by the following equation:

$\alpha=-3.85 \times 10^{-6}-0.032 \times 10^{-6} \times T+1.734 \times 10^{-11} \times T^{2}+1.49 \times 10^{-15} \times T^{3}$

To complete our mathematical model, the initial and boundary conditions are used:

$\underline{\text { Initial conditions: }}$

$\forall t<t_{0}$

$T=T_{0}=273.15 \mathrm{~K}$

$V=V_{0}=0 \mathrm{~V}$

Boundary conditions:

$\forall \mathrm{t}>\mathrm{t}_{0}$

At $x=0, \forall y: T=T_{c}=T_{0}$

At $x=x_{\max }, \forall y: T=T_{h}$

At $y=0, \forall x$ : the graphite sheet is considered adiabatic $Q=\left(\frac{d T}{d x}\right)_{y=0}=0$

At $y=y_{\max }, \forall x$ : the graphite sheet is considered adiabatic $Q=\left(\frac{d T}{d x}\right)_{y=y_{\max }}=0$

\section{Numerical Techniques}

Two numerical techniques are used to scheme our mathematical model in order to be correctly analyzed. These two methods are the explicit finite difference method and the finite element method.

\section{Finite difference scheme}


The heat equation in the graphite sheet is schemed using the explicit form of the finite difference method. The resulting scheming equation is represented by the following form:

$T_{(i, j)}^{n+1}=\left[M K_{(i+1, j)}^{n}-\frac{P T_{(i, j)}^{n}}{\rho C p \Delta x}\right] T_{(i+1, j)}^{n}+\left[1-K_{(i, j)}^{n}(2 M+2 N)+\frac{\Delta t}{\rho C p} P T_{(i, j)}^{n}\left(\frac{1}{\Delta x}+\right.\right.$ $\left.\left.\frac{1}{\Delta y}\right)\right] T_{(i, j)}^{n}+M K_{(i-1, j)}^{n} T_{(i-1, j)}^{n}+\left[N K_{(i, j+1)}^{n}-\frac{P T_{(i, j)}^{n}}{\rho C p \Delta y}\right] T_{(i, j+1)}^{n}+N K_{(i, j-1)}^{n} T_{(i, j-1)}^{n}+$ $\frac{\Delta t}{\rho C p} \frac{J_{(i, j)}^{2 n}}{\sigma_{(i, j)}^{n}}$

With $=\frac{\Delta t}{\rho C p \Delta x^{2}} ; N=\frac{\Delta t}{\rho C p \Delta y^{2}}$ and $P T_{(i, j)}^{n}=\tau_{(i, j)}^{n} J_{(i, j)}^{n}$

The preliminary stability study of the program leads to a scheme of $100 \times 100 \times 60001$ with a time step $\square t=0.0006 \mathrm{~s}$.

\section{Finite Element scheme}

The mathematical model is also solved using the finite element method as reported by Dgheim (Dgheim, 2015, 2016). Thus, the formulation of the weak integral of the thermoelectric problem is obtained as the following:

$$
w\left(T, T^{*}\right)=\int_{V} T^{*} \rho C_{P} \dot{T} d V-\int_{V} T^{*} \nabla \cdot(k \nabla T) d V-\int_{V} T^{*}\left(\frac{J^{2}}{\sigma_{T}}-\tau J \nabla T\right) d V=0
$$

The finite element approximation of the equation (12) can be written as:

$$
[D] \frac{\{T\}^{n+1}-\{T\}^{n}}{\Delta t}+([C]-[H]-[M])\{T\}^{n}+[L]=0
$$

Where, $[D]$ is the storage matrix, $[C]$ is the conductive matrix on a volume, $[H]$ is the conductive matrix in a surface, $[M]$ is the Thomson vector and $[L]$ is the constant matrix.

Then, equation (13) is solved using an explicit finite difference method and Gauss-Legendre integration.

\section{RESULTS AND DISCUSSION}


The numerical model is performed for graphite, for variables thermal conductivity and Seebeck coefficient.

\section{Model Accuracy}

The computation is performed to determine the evolution of the voltage difference versus temperature difference of the graphite sheet. This evolution shows that the voltage difference increases linearly with the increasing of the temperature difference applied at the two edges of the used material.

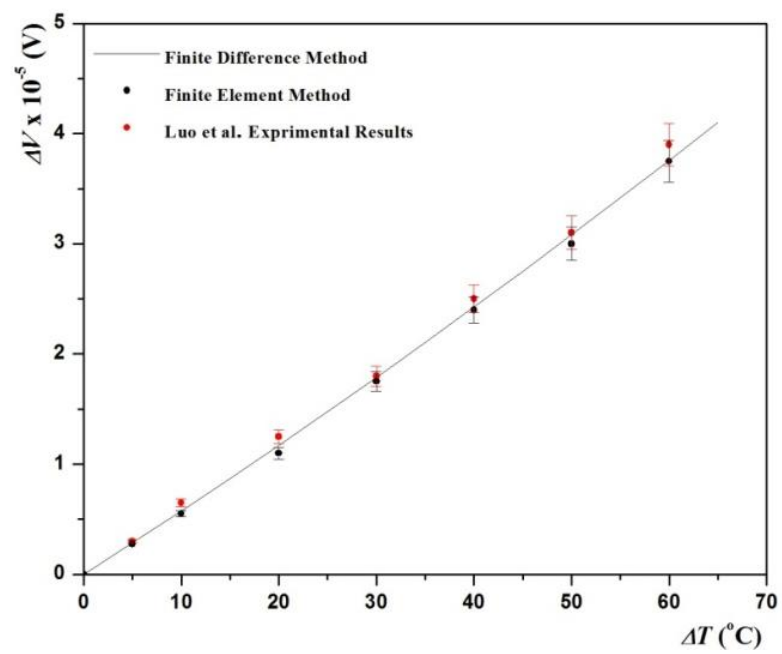

Figure 2. Comparison between our numerical results and Luo et al. experimental results.

The results of our numerical model using the finite difference scheme for a graphite sheet (Figure 2) are compared, under the same conditions, with the ones of our model using the finite element scheme and with the experimental results obtained by Luo et al. (Luo et al., 2002). Good qualitative and quantitative agreements are observed between the whole results. The relative error is less than $10 \%$.

\section{Numerical Results}

The evolution of the voltage difference for different temperature gradients and for two samples of graphite sheets is represented in Figure 3. For the same sample of graphite sheet, one shows that by increasing the gradient temperature, the absolute value of the voltage difference increases, to reach a maximum value of $30.8 \square \mathrm{V}$ at a sheet length $x=0.026 \mathrm{~m}$. When the linear dimension of the graphite sheet becomes longer, the absolute values of the voltage difference becomes less important compared to smaller graphite sheet. This phenomenon is due to the thermo-physical properties of the 
material that depend on the size and the shape of the material structural features. Thus, the thermo-physical properties influence on the temperature distribution in the graphite sheet that modifies the values of the voltage difference.

The voltage profiles are found to be optimum for a structure of $0.026 \times 0.025 \mathrm{~m}^{2}$. For small sizes less than $0.026 \times 0.025 \mathrm{~m}^{2}$, the heat is transferred quickly from the hot boundary to the cold one, which increases rapidly the thermal conductivity and the absolute values of the voltage differences. This phenomenon has the risk to modify the atomic structure of the material. Inversely, when the structure size is superior to $0.026 \times 0.025 \mathrm{~m}^{2}$, the heat resistance is increased, that leads to an augmentation of the electrical resistivity and a reduction of the electrical current.

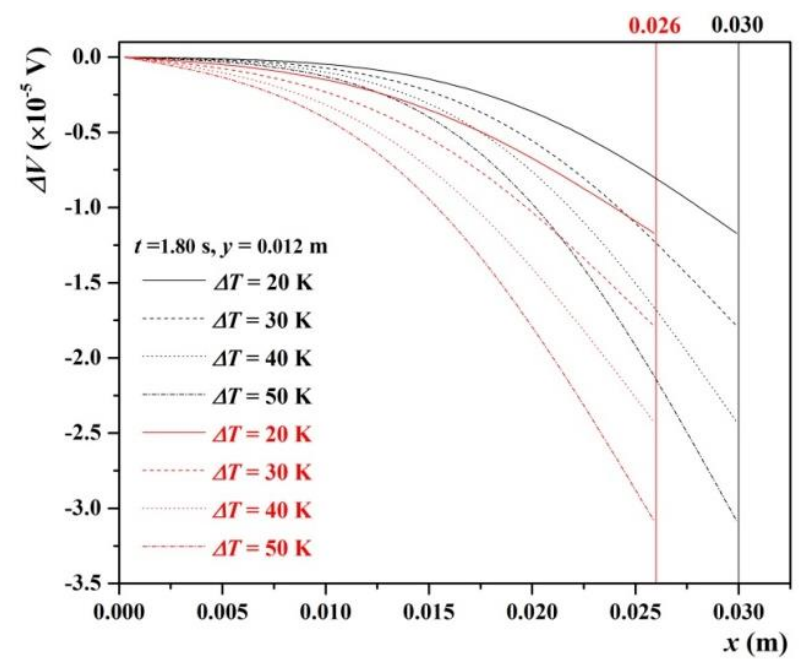

Figure 3. Voltage evolution for different temperature differences for two sample dimensions.

In figure 4 , the absolute value of the voltage difference increases with time to reach its saturation value equal to $7.6 \square \mathrm{V}$ after $18.8 \mathrm{~s}$. In addition, the augmentation of the temperature versus time increases the absolute value of the voltage difference. 


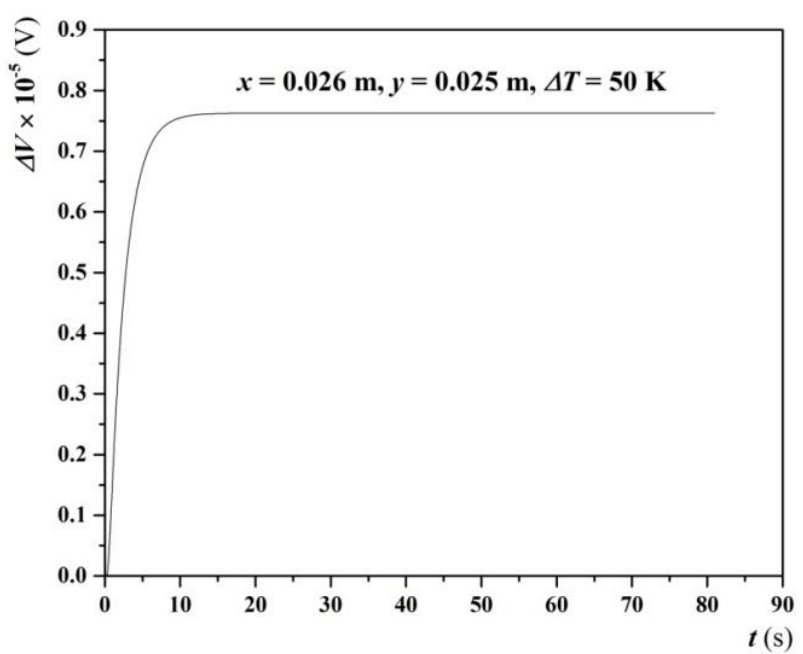

Figure 4. Time evolution of the voltage difference.

In figure 5, the temperature evolution versus $x$ axis is represented for different times at a position $y=0.025 \mathrm{~m}$ and for a temperature difference of $50 \mathrm{~K}$ applied at the edges of the graphite sheet. The applied gradient temperature improves the heat exchange to be transferred from the hot medium to the cold one. Then, the temperature increases from its cold value to reach its hot one. Thus, free carriers will diffuse from the hot side to the cold one. Therefore, a potential difference is generated between the two edges. The potential difference depends on the Seebeck coefficient and the temperature difference of the used material.

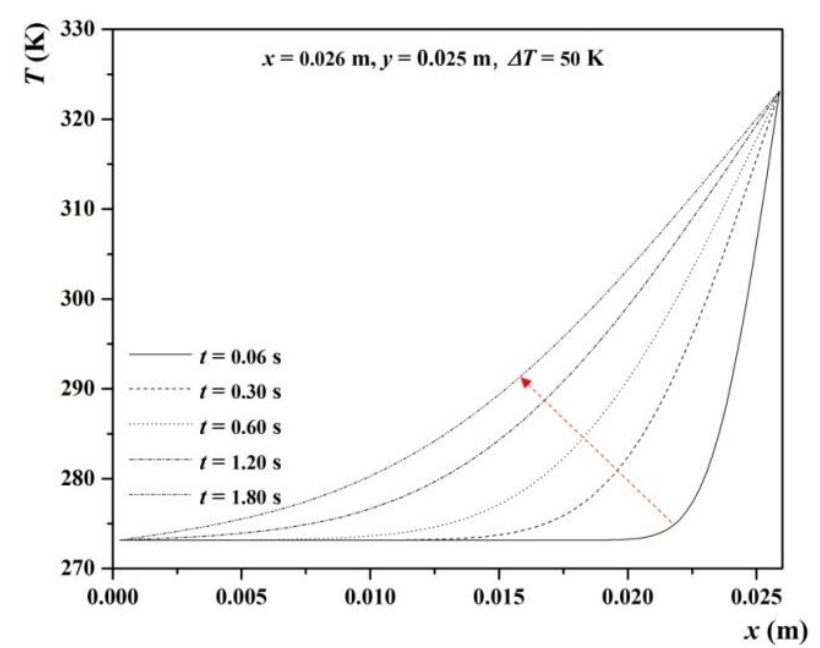

Figure 5. Temperature evolution for different times. 
Otherwise, a slight increase in the temperature gradient influences the Seebeck coefficient as shown in figure 6. The high values of the Seebeck coefficient lead to the displacement of both charges and carriers towards the cold edge. This phenomenon cancels the induced Seebeck voltage. Thus, low carrier concentrations have large Seebeck coefficient. By assuming the electrons in a metal to the molecules in a gas, the electrons tend to diffuse faster when a metal is hotter. This phenomenon makes the hotter edge slightly positively charged and the colder one slightly negatively charged, producing a voltage difference.

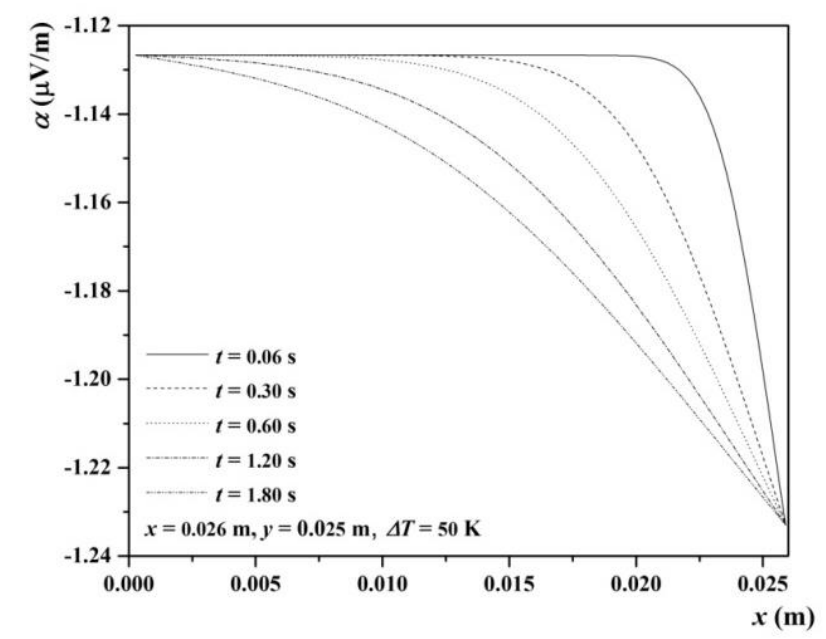

Figure 6. Seebeck coefficient evolution for different times.

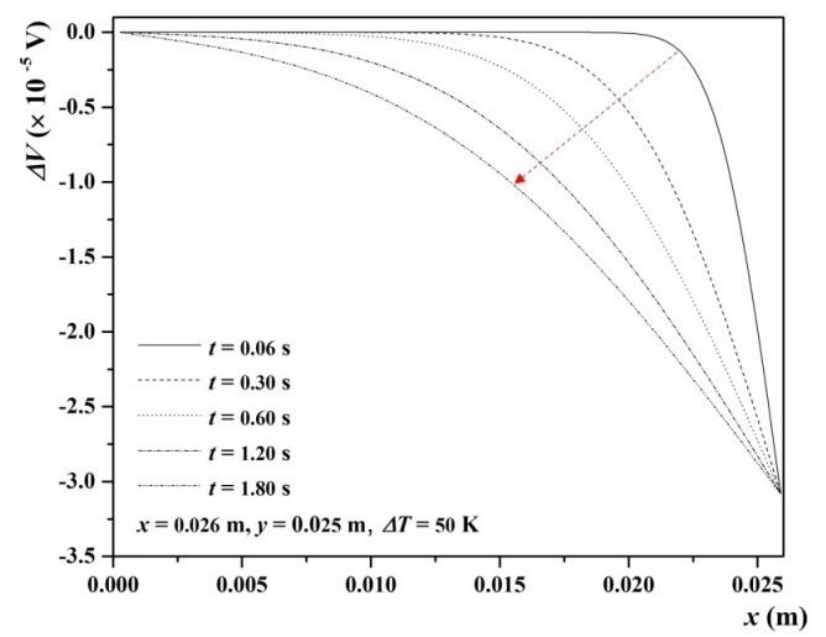

Figure 7. Voltage evolution for different times. 
The absolute value of the voltage difference increases with the time and along the length of the material (figure 7) due to the increasing of the kinetic energy of the carriers. When the carrier concentrations grow up, the Seebeck coefficient falls down, that maximizes the figure of merit to a certain value of temperature equal to $850 \mathrm{~K}$, belong this value, the figure of merit decreases showing an augmentation of the Seebeck coefficient. The computed maximum value of the figure of merit is equal to 1.27 (figure 8). This is an optimization value of the thermoelectric figure of merit of the graphite sheet corresponding to a large thermo-power, high carrier concentrations, and low thermal conductivity as these transport characteristics depends on correlated material properties.

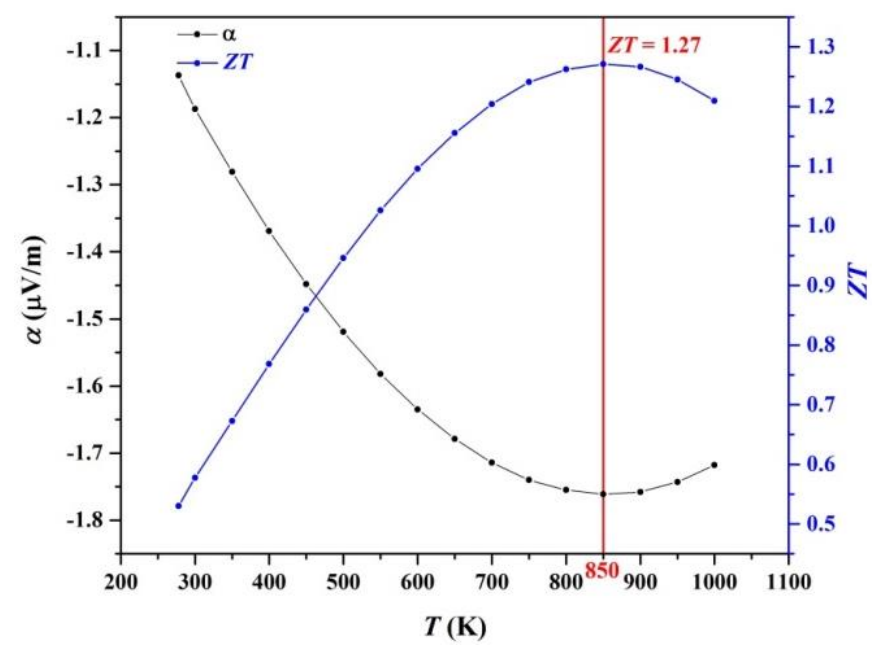

Figure 8. Seebeck coefficient and figure of merit evolution for different temperatures.

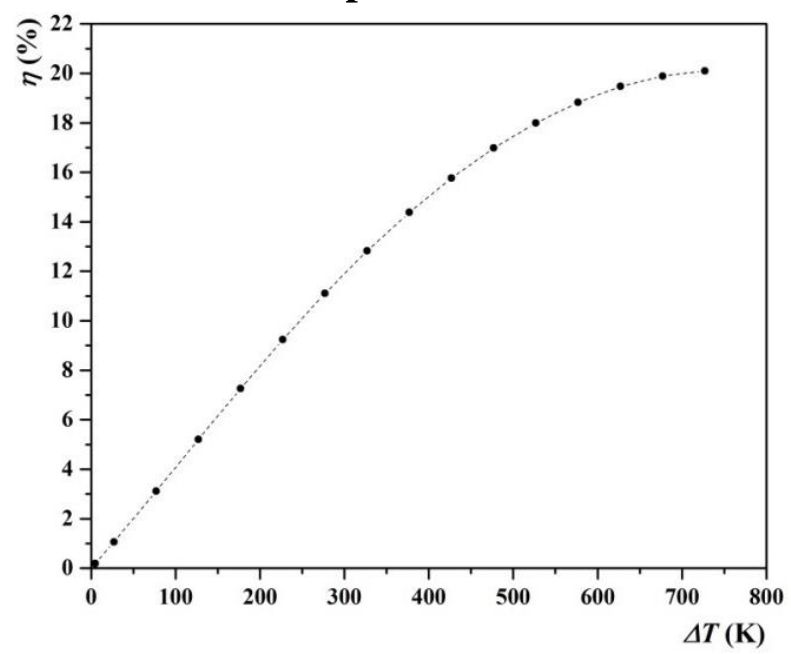

Figure 9. Efficiency evolution for variable temperature differences. 
The maximum efficiency coefficient of the thermoelectric device is temperature dependent. When the temperature difference increases, the maximum efficiency also increases (figure 9). For large temperature differences, the maximum efficiency is high because the Carnot efficiency that it depends on, becomes important. Otherwise, the maximum efficiency highly depends on the figure of merit ZT. For a large $\square T$, the electrical current required for highest efficiency operation changes as the materials properties change with temperature or time.

\section{CONCLUSION}

A numerical model describing the heat conversion into electricity developed in the thermoelectric materials such as graphite is determined. Our mathematical model introduces the temperature difference applied on the edges of the sample to treat the electric properties of the graphite sheet. The results of our numerical techniques are compared with the experimental results of Luo et al. and good qualitative and quantitative agreements are observed. The obtained results are as follows:

The influence of the graphite sheet dimension on the temperature and voltage is studied. An optimal dimension of $0.026 \times 0.025 \mathrm{~m}^{2}$ is considered.

The absolute value of Seebeck coefficient rises up, that maximizes the figure of merit, to reach a maximum value $Z T_{\max }=1.27$ at a temperature of $850 \mathrm{~K}$. After this value, the figure of merit decreases showing an augmentation of the Seebeck coefficient.

The maximum efficiency increases with the increasing of the temperature difference, to reach its maximum value corresponding to $21 \%$.

\section{ACKNOWLEDGMENT}

This work is supported by a grant from the Lebanese University.

\section{REFERENCES}

Hochbaum, A, Chen, R., Delgado, R., Liang, W., Garnett, E., Najarian, M., Majumdar, A. and Yang, P. 2008. Enhanced thermoelectric performance of rough silicon nanowires. Nature, 451: 163-167.

Harman, T., Taylor, P, Walsh, M. and LaForge, B. 2002. Quantum dot super lattice thermoelectric materials and devices. Science, 297: 2229-2232.

Sootsman, J., Chung, D. and Kanatzidis, M. 2009. New and old Concepts in thermoelectric materials. Angewandte Chemie International Edition English, 48: 8616-8639.

Minnich, A., Dresselhaus, M., Ren, Z. and Chen, G. 2009. Bulk nanostructured thermoelectric materials: current research and future prospects. Energy and Environment Science, 2: 466-479. 
Rowe, D. 1995. Handbook of Thermoelectrics. CRC Press, Boca Raton, FL. 720 pp.

Radhakrishnan, R. 2008. A Review of: Thermoelectric Handbook, Macro to Nano. Materials and Manufacturing Processes, 23: 626-627.

Snyder, G. and Toberer, E. 2008. Complex thermoelectric materials. Nature Materials, 7: 105-114.

Kanatzidis, M. 2010. Nanostructured thermoelectrics: The New Paradigm?. Chemistry of Materials, 22: 648-659.

Gayner, C. and Kar, K. 2016. Recent advances in thermoelectric materials. Progress in Materials Science, 83: 330-382.

Kumar, S., Heister, S. D., Xu, X., Salvador, J. R. and Meisner, G. P. 2013. Thermoelectric generators for automotive waste heat recovery systems part I: numerical modeling and baseline model analysis. Journal of Electronic Materials, 42: 665-674.

Shi, Y., Wang, Y., Mei, D. and Chen, Z. 2018. Numerical modeling of the performance of thermoelectric module with polydimethylsiloxane encapsulation. International Journal of Energy Research, 42: 1287-1297.

Brus, V., Gluba, M., Rappich, J., Lang, F., Maryanchuk, P. and Nickel, N. 2018. Fine art of thermoelectricity. Applied Materials and Interfaces, 10: 4737-4742.

Luo, X., Chugh, R., Biller, B., Hoi, Y. M. and Chung, D. L. 2002. Electronic applications of flexible graphite. J. Electronic Materials, 31: 535-544.

Klein, C. A. and Donadio, R. N. 1967. The seebeck coefficient of graphite or the STB model revisited. $8^{\text {th }}$ Biennial Conference-BUFFALO, NY, p. 29.

Dgheim, J. 2015. Numerical analysis of the heat transfer in heterojunction device for optoelectronic applications. Journal of Applied and Computational Mathematics, 04. http://dx.doi.org/10.4172/2168-9679.1000264

Dgheim, J. 2016. Modeling of UV laser beam silicon nitride interaction. Laser Physics, 26:115001. http://dx.doi.org/10.1088/1054-660X/26/11/115001 